\title{
ADAPTIVE DECODING FOR HALFTONE ORIENTATION-BASED DATA HIDING
}

\author{
Orhan Bulan, Gaurav Sharma \\ ECE Dept., University of Rochester, \\ Rochester, NY, 14627-0126, USA
}

\author{
Vishal Monga \\ Xerox Research Center Webster \\ Webster, NY, 14580, USA
}

\begin{abstract}
Halftone image watermarking techniques that allow automated extraction of the embedded watermark data are useful in a variety of document security and workflow applications. The print-scan process inherent in these applications introduces distortions whose characteristics exhibit a strong spatial dependence on the cover image in which the data is embedded. In this paper, we demonstrate that a characterization of this channel dependence and the use of error correction coding that exploits this dependence via image adaptive decoding offers a significant performance gain. We show this advantage in the specific context of a high rate data embedding method that utilizes orientation modulation for data embedding in clustered dot halftones and moment based detection at the receiver. Channel coding for this scenario utilizing convolutional codes and Repeat Accumulate (RA) codes highlights the advantage of the proposed adaptive decoding methodology. The performance for the RA codes with adaptive decoding also reveals that the resulting system has significantly higher operational rates than prior schemes.
\end{abstract}

Index Terms - Image adaptive decoding, data hiding

\section{INTRODUCTION}

Hardcopy data embedding techniques that allow automated extraction of the embedded data without involving a human observer are a key enabler for a wide variety of applications. Methods developed for this purpose can be categorized as data encoding or data hiding approaches. For methods in the former category, the data is embedded in a region of the printed page that is solely dedicated to the objective of conveying the information and the visual appearance of the encoded region is only of secondary concern. One and two-dimensional bar-codes are the pre-dominant representatives of techniques in this class. Methods for data hiding in hardcopy documents, on the other hand, must carry the embedded information in manner that minimally disrupts the primary content included in the print, while still allowing recovery of the embedded data at the receiver. This is a challenging constraint particularly when the primary content is an image, which is the situation we consider in this paper.

Conventional image data embedding methods that are robust to a print-scan attack can be employed for embedding information in hardcopy images $[1,2]$. Since these methods do not particularly exploit the characteristics of the specific printing process employed, methods that exploit this information can offer significant performance improvements in terms of robustness and embedding rates. In recent work [3], we proposed one such method for clustered-dot halftone printing by utilizing elliptically shaped halftone dots and

This work was supported in part by Xerox Corporation and by a grant from New York State Office of Science, Technology and Academic Research (NYSTAR) through the Center for Electronic Imaging Systems (CEIS). embedding data in the dot orientation. Though the method has a high embedding rate, the interaction of the halftoning with the cover image content poses a significant challenge. The requirement for a faithful reproduction of the cover in particular can have irreconcilable conflicts with the ability to introduce a distinguishable change in orientation. As a specific example, in regions where the cover image is white, the desired rendering is white paper free of any printed dots, in which situation no discernible orientation can be incorporated. A similar conflict arises in regions where the cover image is black. In these scenarios, as is desirable for data hiding methods, the method proposed in [3] concedes embedding robustness in favor of cover image fidelity ${ }^{1}$. It is also obvious from the preceding discussion, that this dependence of the spatially-localized characteristics of the halftone data hiding channel on the gray-level of the cover image is in fact universal and not merely an artifact of our embedding scheme.

In this paper, we address this challenge by characterizing the dependence of the halftone data embedding channel on the local gray-level of the cover image and introducing channel coding with image adaptive decoding at the receiver that incorporates the dependence in the decoding process. We consider both convolutional codes and near-capacity achieving ${ }^{2}$ RA codes $[5,6]$, both across a range of code-rates. In order to illustrate the benefit of the coverimage dependent channel characterization and the image adaptive decoding, for the convolutional codes, we consider the performance under soft-decoding utilizing the channel characterization and under hard-decoding which disregards this information.

We experimentally evaluate the proposed scheme on a Xerographic printer, first characterizing the cover image dependence of the channel and then evaluating decoding performance over test prints. Our results for the convolutional codes reveal that the use of the image adaptive decoding with the channel characterization yields much lower bit error rates than the hard decoding alternative which does not utilize this characterization. The results obtained with the RA codes also validate the benefit of utilizing embedding schemes and decoding methods that are specifically designed for the print-scan channel in consideration - (operational) error-free embedding rates achieved by our scheme are much greater than those reported by existing hardcopy data hiding methods.

\section{SYSTEM OVERVIEW}

A block diagram summarizing our overall proposed scheme, is shown in Fig. 1. Here $\mathbf{m}$ denotes the message bits to be embedded

\footnotetext{
${ }^{1}$ DataGlyphs ${ }^{\mathrm{TM}}$ [4], which also utilize orientation for data embedding, on the other hand exercise the trade-off in the other direction and therefore are closer to data-encoding rather than data-hiding approaches.

${ }^{2}$ The near capacity achieving moniker is strictly applicable only in the case of an AWGN channel.
} 
and the cover image in which the data is to be embedded is denoted by $I(x, y)$. In order to allow error recovery at the receiver, the channel encoder introduces redundancy in the message producing the coded data c. Using the cover image and the coded data, the operations of halftoning and data embedding are performed jointly in order to minimize the impact on image quality. The resulting image is printed and at the receiver a scan $I_{s}(x, y)$ of the hardcopy image is obtained. Detection statistics are obtained from the scanned image in the form of image moments $\sigma_{x}, \sigma_{y}$ and an estimate $\hat{g}$ of the local gray level. These are utilized by the channel decoder to obtain an estimate $\hat{\mathbf{m}}$ of the transmitted message, either via soft decoding that utilizes a statistical model for the channel or via hard decoding. In the latter case, the moments are first utilized to make a hard decision to obtain an estimate of each individual transmitted bit in $\mathbf{c}$, the collection of these estimated bits is then used in the error correction decoder for the purpose of estimating the message.

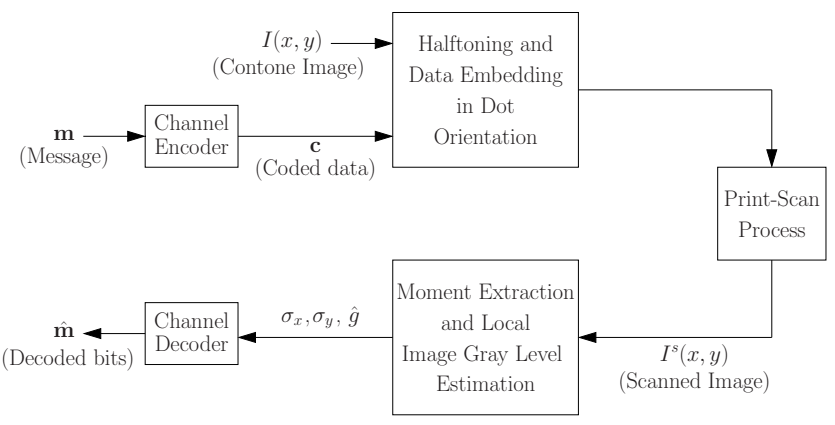

Fig. 1. Block diagram for orientation based data hiding with channel coding.

We describe the individual system elements in detail in the following sections. Note that our primary objective here is to characterize the channel statistical behavior as a function of the local gray-level of the cover image and to exploit this characterization in adaptive decoding at the receiver in order to improve the system performance. Accordingly, in the soft-decision case, the estimated local image gray level at the receiver is utilized in the decoding process ${ }^{3}$.

\section{ORIENTATION MODULATION CHANNEL MODEL INCORPORATING COVER IMAGE DEPENDENCE}

For the purpose of embedding we utilize a method for data hiding in clustered halftone dots using orientation modulation in the halftoning process that we recently introduced [3]. Figure 2 illustrates the embedding method and its characteristics using a sample cover image $e^{4}$. Elliptically shaped halftone dots are utilized with a vertical/horizontal orientation for the major axis depending on value of the embedded bit. Black elliptical dots along the two orientations are produced on a predominantly white background in highlights, i.e. regions of the cover image with less than $50 \%$ halftone area coverage (see Fig. 2 (b)) and white elliptical "holes" with the two possible orientations are produced in the shadows, i.e. regions of the cover image with more than 50\% halftone area coverage (see

\footnotetext{
${ }^{3}$ We recognize that in accordance with informed-embedding principles the embedding should also be adapted to the cover image. This is, however, deferred to future work due to the multiple formidable challenges it introduces in the case of hardcopy channels.

${ }^{4}$ We focus on highlighting the channel dependence on the cover image here and refer the interested reader to [3] for additional details.
}

Fig. 2 (c)). At the receiver, following global and local synchronization, the modulated orientation is detected based on two orthogonal statistically motivated moments computed within each halftone cell from the scan $I^{s}(x, y)$ of the printed image. The moment along the $X$-axis is calculated as:

$$
\sigma_{x}=\frac{1}{\mathcal{S}} \sum_{x, y \in C} I^{s}(x, y)(x-\bar{x})^{2}
$$

where $\mathcal{S}=\sum_{x, y \in C} I^{s}(x, y)$ and $\bar{x}=\frac{1}{\mathcal{S}} \sum_{x, y \in C} I^{s}(x, y) x$ represents the abscissa of the center of mass of the halftone dot. The moment $\sigma_{y}$ along the $Y$-axis is defined and computed in a similar fashion.

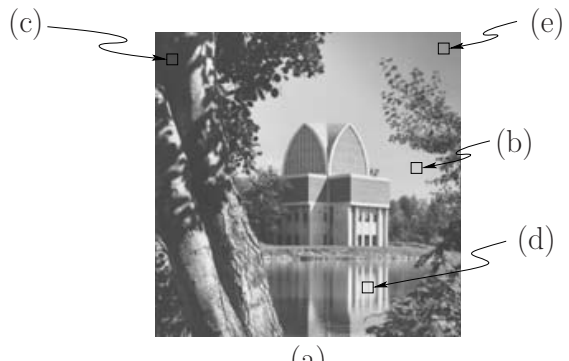

(a)

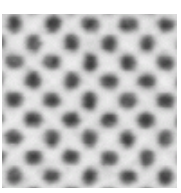

(b)

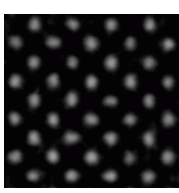

(c)

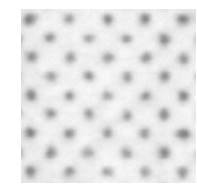

(d)

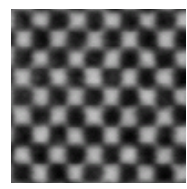

(e)
Fig. 2. Orientation based halftone data embedding.

The embedding scheme is well matched to the characteristics of halftone printing and causes only a minimal increase in the distortion pre-existing in halftones. This is because the halftone reproduction process relies on matching the average gray level of the print to the desired image and the the change in dot orientations induced by the embedding does not change the average gray level within each halftone cell. While the embedding distortion characteristics of the method are quite good, due to the inherent constraints of halftone reproduction, the robustness of the embedding demonstrates significant spatial variability depending on the local cover image content. This is demonstrated in parts (b)-(e) of Fig 2. These figures show enlarged local views from a scan of a print of the cover image of Fig 2 (a) which was generated with data embedding using the halftone orientation modulation technique. The regions of the cover image, to which these local views correspond, are indicated by the alphabetic labels in Fig 2 (a). In regions where the cover image is white, there is no printing and hence clearly the computed moments have no discernible dependence on the embedded message bit value. This limitation also arises in regions where the cover image is close to white as illustrated in Fig 2 (d). Likewise, in regions where the cover image is black, the halftone cells in the print are completely black. Consequently, once again the moments at the receiver convey no useful information about the embedded bit in regions where the cover image is black or near black. Finally, in mid-gray regions of the cover image that correspond to $50 \%$ halftone area coverage, and in regions where the area coverage is close to $50 \%$, the printed halftones takes the configuration of a checker-board pattern and orientation modulation is ineffective (Fig 2 (e)). This impact of the 
gray-level of the cover image on the orientation modulation embedding channel can also be formalized in terms of a gray-level dependent characterization of the channel capacity that we have performed in related recent work [7].

To incorporate the channel dependence on the local gray level of the cover image in our error recovery scheme, we propose a corresponding statistical model for the channel, expressed in the form of the conditional density function $f\left(\sigma_{x}, \sigma_{y} \mid \Theta_{i}, g\right)$ where received moments are conditioned on the local image gray level $g$ and, horizontal and vertical orientations $\Theta_{i}, i=1,2$. The moments along the two orthogonal directions can be seen to be approximately conditionally independent given the embedding orientation and the average gray level ${ }^{5}$. The conditional independence implies that the joint conditional density can be expressed as:

$$
f\left(\sigma_{x}, \sigma_{y} \mid \Theta_{i}, g\right)=f\left(\sigma_{x} \mid \Theta_{i}, g\right) f\left(\sigma_{y} \mid \Theta_{i}, g\right)
$$

which results in the simplified channel model shown in Fig. 3.

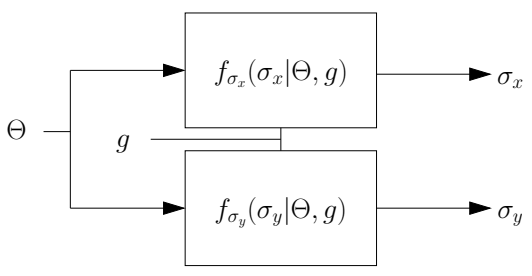

Fig. 3. Probabilistic model for the channel.

We characterized the conditional densities $f\left(\sigma_{x} \mid \Theta_{i}, g\right), f\left(\sigma_{y} \mid\right.$ $\left.\Theta_{i}, g\right)$ in terms of experimentally determined empirical histograms. The kurtosis [8], which is widely used as a shape descriptor parameter for distributions, ranged from approximately 2.7 through 3.5 for these empirical histograms, which was close to the Gaussian case (which has a kurtosis of 3). For mathematical simplicity, we therefore approximated the conditional densities as Gaussians, i.e., $f\left(\sigma_{x} \mid \Theta_{i}, g\right) \sim \mathcal{N}\left(\mu_{x, g}^{i}, s_{x, g}^{2}\right), f\left(\sigma_{y} \mid \Theta_{i}, g\right) \sim \mathcal{N}\left(\mu_{y, g}^{i}, s_{y, g}^{2}\right)$, where $\mu_{x, g}^{i}$ is the mean and $s_{x, g}$ the standard deviation of $f\left(\sigma_{x}\right.$ $\left.\Theta_{i}, g\right)$, and other terms are defined similarly. The channel parameters corresponding to these conditional means and standard deviations were estimated from experimental data by using ExpectationMaximization [9].

\section{ERROR CORRECTION CODING AND DECODING}

\subsection{Channel Coding}

For the channel coding, we consider two code families viz. convolutional codes and repeat accumulate (RA) codes [5,6]. The choice of convolutional codes was motivated by the fact that good codes with low/moderate complexity are known for a variety of rates [10] and the merit of the probabilistic channel model can be clearly demonstrated for these codes by contrasting soft and hard decoding. The choice of RA codes was motivated by the fact that they are near capacity-achieving $[5,6]$ and also readily allow the rate to be varied through a change of the repetition parameter.

${ }^{5}$ This was experimentally validated in prior work [3] for constant gray level images.

\subsection{Channel Decoding}

Maximum likelihood decoding of convolutional codes can be achieved by the Viterbi algorithm [11] which allows for both hard and soft decoding. Approximate maximum aposteriori probability (MAP) decoding for RA codes is accomplished using belief propagation [12] for iterative decoding.

\subsubsection{Hard Decoding}

The hard decoding process is a concatenation of "hard detection" followed by classical error correction hard decoding. . Since the embedding process orients the dots along $X$ or $Y$ axes, which are coincident with the two orthogonal directions along which the moments $\sigma_{x}$ and $\sigma_{y}$ are computed (see Section 3), we use the intuitively motivated, albeit heuristic, hard detection rule

$$
\sigma_{x} \underset{0}{\gtrless} \sigma_{y} .
$$

to decode individual bits. Error correction decoding is then performed based on extracted bits.

\subsubsection{Soft Decoding}

As is evident here, and also well known from classical communications, hard decoding does not exploit the knowledge of probabilistic channel model. Soft decoding can offer performance improvements by propagating the knowledge of the channel as a soft measure to the error correction decoder. This is illustrated in Fig. 4. For both convolutional and RA codes, the soft measure used in decoding is the log likelihood ratio:

$$
\gamma=\log \frac{p\left(\Theta_{1}, \hat{g}\right)}{p\left(\Theta_{2}, \hat{g}\right)}
$$

where $p\left(\Theta_{i}, \hat{g}\right)$ is the likelihood given by

$$
p\left(\Theta_{i}, \hat{g}\right)=f_{\sigma_{x}}\left(\sigma_{x} \mid \Theta_{i}, \hat{g}\right) \cdot f_{\sigma_{y}}\left(\sigma_{y} \mid \Theta_{i}, \hat{g}\right)
$$

where $\sigma_{x}, \sigma_{y}$ are the image moments along the $X$ and $Y$ directions computed within the halftone cell as indicated in Section 3 and $\hat{g}$ is the estimate of the local graylevel obtained as the average of the pixel intensity values within the halftone cell.

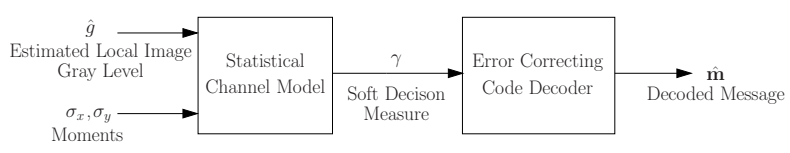

Fig. 4. Image Adaptive Soft Decoding.

\section{EXPERIMENTAL RESULTS}

We implemented our proposed halftone image data embedding scheme and the associated image adaptive decoding and evaluated its performance experimentally utilizing a print-scan channel consisting of a xerographic printer and a desktop scanner. A randomly generated bit stream was used as the message data in our experiments. This data was encoded utilizing the encoder for the error correction code in consideration (either convolutional or RA). The resulting coded data and a target cover image served as the input 
to a module which jointly performed the halftoning and the dataembedding operations. In our work we utilized a $45^{\circ}$ orthogonal halftone screen ${ }^{6}$ with a frequency of 75 cells per inch. The resulting halftone image (with data embedded in the orientation) was printed on the Xerographic printer, which had an addressability of 2400 dots per inch (dpi). The resulting print was scanned on a desktop scanner with a 1200 dpi resolution. Global synchronization was performed by utilizing the periodic grid of the halftones and geometric distortion in the printing process was accounted for via decision directed local synchronization (See [3] for details). Post-synchronization, the estimate $\hat{g}$ of the average gray-level in the halftone cell was obtained and image moments $\sigma_{x}$ and $\sigma_{y}$ along the $X$ and $Y$ axes, respectively, were computed as indicated in Section 3.

The print-scan channel was first characterized by utilizing a set of training images, from which the the parameters for the statistical channel model of Section 3 were obtained via the EM algorithm [9]. The system performance was then evaluated by utilizing an independent test image, specifically the Chapel image shown in Fig. 2 (a). The printed size of the image was $8 \times 8$ inches and a total of 414960 coded bits were embedded in the resulting printed image.

When using convolutional codes, the coded information bits (and corresponding halftone images with data embedding) were generated for several different code rates denoted by $1 / n$, where $n=2,3,4,5,6,7,8,10$, and 12 . The convolutional codes used in our experiments are among the best codes known with low constraint length [10]. Fig. 5 plots decoding results in the form of bit error rate (BER) as a function of the code rate. As is evident from Fig. 5, soft decoding enabled by the cover dependent channel model significantly reduces bit errors. This benefit is particularly pronounced at low code-rates.

As with convolutional codes, we generate several different halftone images with embedded data that was generated using RA coding on message bits. From scans of these printed images, we determined that a code rate of $1 / 4$ allows for error free decoding. This rate hence, provides a lower bound on data hiding capacity, that is 0.25 bits per halftone cell. This rate comfortably exceeds those reported by existing data hiding methods in the literature $[1,2,14]$ and approaches the capacity of hardcopy data encoding methods $[4,15]$.

\section{CONCLUSION}

We develop a high-rate data hiding method for clustered-halftone printed images, with automated recovery of embedded data. The primary technical problem we address in this paper is adapting to the dependence of the print-scan channel on the cover image content through a suitable probabilistic channel model which is then utilized for adaptive decoding at the receiver. Experiments conducted on xerographic printers confirm the merits of our proposed channel and image adaptive decoding. First, for the convolutional codes, softdecoding using the print-scan channel model significantly reduces bit-error rates in comparison with hard decoding. Second, using RA codes with the adaptive decoding, our scheme achieves error-free embedding rates that are significantly larger than those achieved by prior methods.

\section{REFERENCES}

[1] K. Solanki, U. Madhow, B. S. Manjunath, S. Chandrasekaran, and I. ElKhalil, "Print and scan resilient data hiding in images," IEEE Trans. Info. Forensics and Security, vol. 1, no. 4, pp. 464-478, Dec. 2006.

${ }^{6}$ The orientation of the screen [13, Chap 6] is not to be confused with the orientation of the halftone dots used for embedding.

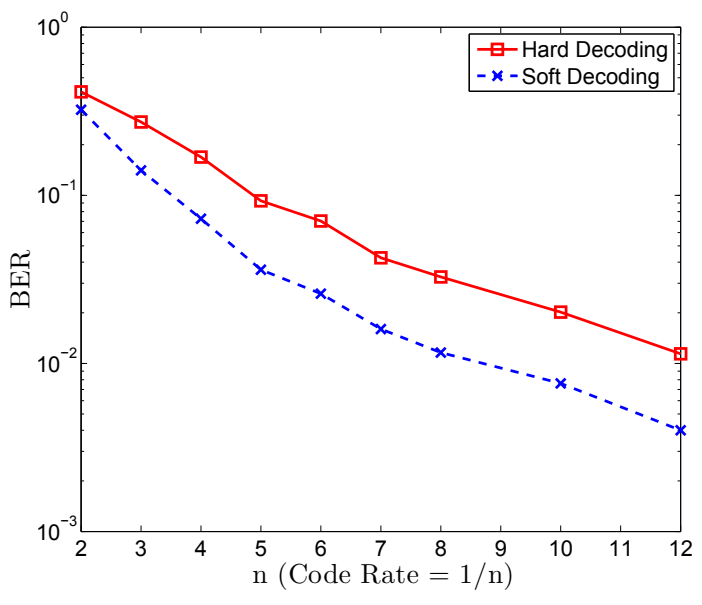

Fig. 5. Soft and hard decoding performances with convolutional codes over various code rates.

[2] D. Corp, "Digimarc mediabridge," 2000. [Online]. Available: www.digimarc.com/tech/docs/dmrc_media_bridge.pdf

[3] O. Bulan, V. Monga, G. Sharma, and B. Oztan, "Data embedding in hardcopy images via halftone-dot orientation modulation," in Proc. SPIE: Security, Forensics, Steganography, and Watermarking of Multimedia Contents X, E. J. Delp, P. W. Wong, J. Dittmann, and N. D. Memon, Eds., vol. 6819, Jan. 2008, pp. 68 190C-1-12.

[4] D. L. Hecht, "Printed embedded data graphical user interfaces," IEEE Computer, pp. 47-55, Mar. 2001.

[5] D. Divsalar, H. Jin, and R. J. McEliece, "Coding theorems for "turbolike" codes," in Proc. Allerton Conference, Monticello, IL, USA, Sept. 1998, pp. 201-210

[6] H. Jin, A. Khandekar, and R. McEliece, "Irregular repeataccumulate codes," in Proc. 2nd Intl. Symp. on Turbo codes and Related Topics, Sep. 2000, pp. 1-8. [Online]. Available: citeseer.ist.psu.edu/jin00irregular.html

[7] O. Bulan, G. Sharma, and V. Monga, "On the capacity of orientation modulation halftone channels," in Proc. IEEE Intl. Conf. Acoustics Speech and Sig. Proc., Apr. 2008, pp. 1685-1688.

[8] K. Najim, E. Ikonen, and A.-K. Daoud, Stochastic Processes:Estimation, Optimisation and Analysis. London, UK: Sterling, VA : Kogan Page Science, 2004.

[9] T. K. Moon, "The expectation-maximization algorithm," IEEE Signal Processing Magazine, vol. 13, no. 6, pp. 47-60, 1996.

[10] D. Daut, J. Modestino, and L. Wismer, "New short constraint length convolutional code constructions for selected rational rates," IEEE Trans. Info. Theory, vol. 28, no. 5, pp. 794-800, 1982.

[11] G. D. Forney Jr, "The Viterbi algorithm," in Proceedings of the IEEE, vol. 61, no. 3, 1973, pp. 268-278.

[12] J. Pearl, Probabilistic Reasoning in Intelligent Systems: Networks of Plausible Inference. Morgan Kaufmann, 1988.

[13] G. Sharma, Ed., Digital Color Imaging Handbook. Boca Raton, FL: CRC Press, 2003.

[14] B. Oztan and G. Sharma, "Continuous phase modulated halftones and their application to halftone data embedding," in Proc. IEEE Intl. Conf. Acoustics Speech and Sig. Proc., vol. II, May 2006, pp. 333-336. [Online]. Available: /OztanSharmaCPMHalftoneWMICASSP06.pdf

[15] “Data Matrix barcode FAQ \& tutorial,” accessed Jan 2008. [Online]. Available: http://idautomation.com/datamatrixfaq.html 